\title{
Relaxing planarity for topological graphs ${ }^{\star}$
}

\author{
János Pach ${ }^{1}$, Radoš Radoičić ${ }^{2}$, and Géza Tóth ${ }^{3}$ \\ 1 City College, CUNY and Courant Institute of Mathematical Sciences, \\ New York University, New York, NY 10012, USA \\ pach@cims.nyu.edu \\ 2 Department of Mathematics, \\ Massachusetts Institute of Technology, Cambridge, MA 02139, USA \\ rados@math.mit.edu \\ 3 Rényi Institute of the Hungarian Academy of Sciences, \\ H-1364 Budapest, P.O.B. 127, Hungary \\ geza@renyi.hu
}

\begin{abstract}
According to Euler's formula, every planar graph with $n$ vertices has at most $O(n)$ edges. How much can we relax the condition of planarity without violating the conclusion? After surveying some classical and recent results of this kind, we prove that every graph of $n$ vertices, which can be drawn in the plane without three pairwise crossing edges, has at most $O(n)$ edges. For straight-line drawings, this statement has been established by Agarwal et al., using a more complicated argument, but for the general case previously no bound better than $O\left(n^{3 / 2}\right)$ was known.
\end{abstract}

\section{Introduction}

A geometric graph is a graph drawn in the plane so that its vertices are represented by points in general position (i.e., no three are collinear) and its edges by straight-line segments connecting the corresponding points. Topological graphs are defined similarly, except that now each edge can be represented by any simple (non-selfintersecting) Jordan arc passing through no vertices other than its endpoints. Throughout this paper, we assume that if two edges of a topological graph $G$ share an interior point, then at this point they properly cross. We also assume, for simplicity, that no three edges cross at the same point and that any two edges cross only a finite number of times. If any two edges of $G$ have at most one point in common (including their endpoints), then $G$ is said to be a simple topological graph. Clearly, every geometric graph is simple. Let $V(G)$ and $E(G)$ denote the vertex set and edge set of $G$, respectively. We will make no notational distinction between the vertices (edges) of the underlying abstract graph, and the points (arcs) representing them in the plane.

It follows from Euler's Polyhedral Formula that every simple planar graph with $n$ vertices has at most $3 n-6$ edges. Equivalently, every topological graph with $n$ vertices and more than $3 n-6$ edges has a pair of crossing edges. What happens if, instead of a crossing pair of edges, we want to guarantee the existence of some larger configurations involving several crossings? What kind of unavoidable substructures must occur in every geometric (or topological) graph $G$ having $n$ vertices and more than $C n$ edges, for an appropriate large constant $C>0$ ?

In the next four sections, we approach this question from four different directions, each leading to different answers. In the last section, we prove that any topological graph with $n$ vertices and no three pairwise crossing edges has at most $O(n)$ edges. For simple topological graphs, this result was first established by Agarwal-Aronov-Pach-Pollack-Sharir [AAPPS97], using a more complicated argument.

* János Pach has been supported by NSF Grant CCR-00-98245, by PSC-CUNY Research Award 633520036, and by OTKA T-032458. Géza Tóth has been supported by OTKA-T-038397 and by an award from the New York University Research Challenge Fund. 


\section{$2 \quad$ Ordinary and topological minors}

A graph $H$ is said to be a minor of another graph $G$ if $H$ can be obtained from a subgraph of $G$ by a series of edge contractions (and deletions). If a subgraph of $G$ can be obtained from $H$ by replacing its edges with independent paths between their endpoints, then $H$ is called a topological minor of $G$. Clearly, a topological minor of $G$ is also its (ordinary) minor.

If a graph $G$ with $n$ vertices has no minor isomorphic to $K_{5}$ or to $K_{3,3}$, then by Kuratowski's theorem it is planar and its number of edges cannot exceed $3 n-6$. It follows from an old result of Wagner that the same conclusion holds under the weaker assumption that $G$ has no $K_{5}$ minor. A few years ago Mader [M98] proved the following famous conjecture of Dirac:

Theorem 2.1. (Mader) Every graph of $n$ vertices with no topological $K_{5}$ minor has at most $3 n-6$ edges.

If we only assume that $G$ has no topological $K_{r}$ minor for some $r>5$, we can still conclude that $G$ is sparse, i.e., its number of edges is at most linear in $n$.

Theorem 2.2. (Komlós-Szemerédi [KSz96], Bollobás-Thomason [BT98]) For any positive integer $r$, every graph of $n$ vertices with no topological $K_{r}$ minor has at most $c r^{2} n$ edges.

Moreover, Komlós and Szemerédi showed that the above statement is true with any positive constant $c>1 / 4$, provided that $r$ is large enough. Apart from the value of the constant, this theorem is sharp, as is shown by the union of pairwise disjoint copies of a complete bipartite graph of size roughly $r^{2}$.

We have a better bound on the number of edges, under the stronger assumption that $G$ has no $K_{r}$ minor.

Theorem 2.3. (Kostochka [K84], Thomason [T84]) For any positive integer $r$, every graph of $n$ vertices with no $K_{r}$ minor has at most $c r \sqrt{\log r} n$ edges.

The best value of the constant $c$ for which the theorem holds was asymptotically determined in [T01]. The theorem is sharp up to the constant. (Warning! The letters $c$ and $C$ used in several statements will denote unrelated positive constants.)

Reversing Theorem 2.3, we obtain that every graph with $n$ vertices and more than $c r \sqrt{\log r} n$ edges has a $K_{r}$ minor. This immediately implies that if the chromatic number $\chi(G)$ of $G$ is at least $2 c r \sqrt{\log r}+1$, then $G$ has a $K_{r}$ minor. According to Hadwiger's notorious conjecture, for the same conclusion it is enough to assume that $\chi(G) \geq r$. This is known to be true for $r \leq 6$ (see [RST93]).

\section{Quasi-planar graphs}

A graph is planar if and only if it can be drawn as a topological graph with no crossing edges. What happens if we relax this condition and we allow $r$ crossings per edge, for some fixed $r \geq 0$ ?

Theorem 3.1. [PT97] Let $r$ be a natural number and let $G$ be a simple topological graph of $n$ vertices, in which every edge crosses at most $r$ others. Then, for any $r \leq 4$, we have $|E(G)| \leq(r+3)(n-2)$.

The case $r=0$ is Euler's theorem, which is sharp. In the case $r=1$, studied in [PT97] and independently by Gärtner, Thiele, and Ziegler (personal communication), the above bound can be attained for all $n \geq 12$. The result is also sharp for $r=2$, provided that $n \equiv 5(\bmod 15)$ is sufficiently large (see Figure 1). 


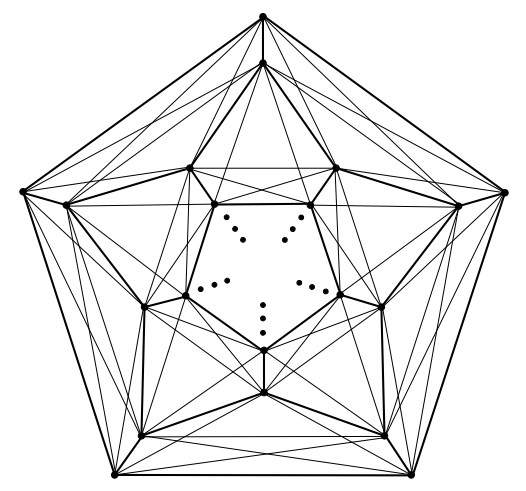

Figure 1.

However, for $r=3$, we have recently proved that $|E(G)| \leq 5.5(n-2)$, and this bound is best possible up to an additive constant [PRTT02]. For very large values of $r$, a much better upper bound can be deduced from the following theorem of Ajtai-Chvátal-Newborn-Szemerédi [ACNS82] and Leighton [L84]: any topological graph with $n$ vertices and $e>4 n$ edges has at least constant times $e^{3} / n^{2}$ crossings.

Corollary 3.2. [PRTT02] Any topological graph with $n$ vertices, whose each edge crosses at most $r$ others, has at most $4 \sqrt{r} n$ edges.

One can also obtain a linear upper bound for the number of edges of a topological graph under the weaker assumption that no edge can cross more than $r$ other edges incident to the same vertex. This can be further generalized, as follows.

Theorem 3.3. [PPST02] Let $G$ be a topological graph with $n$ vertices which contains no $r+s$ edges such that the first $r$ are incident to the same vertex and each of them crosses the other $s$ edges. Then we have $|E(G)| \leq C_{s} r n$, where $C_{s}$ is a constant depending only on s.

In particular, it follows that if a topological graph contains no large gridlike crossing pattern (two large sets of edges such that every element of the first set crosses all elements of the second), its number of edges is at most linear in $n$. It is a challenging open problem to decide whether the same assertion remains true for all topological graphs containing no large complete crossing pattern.

For any positive integer $r$, we call a topological graph $r$-quasi-planar if it has no $r$ pairwise crossing edges. A topological graph is $x$-monotone if all of its edges are $x$-monotone curves, i.e., every vertical line crosses them at most once. Clearly, every geometric graph is $x$-monotone, because its edges are straight-line segments (that are assumed to be non-vertical). If the vertices of a geometric graph are in convex position, then it is said to be a convex geometric graph.

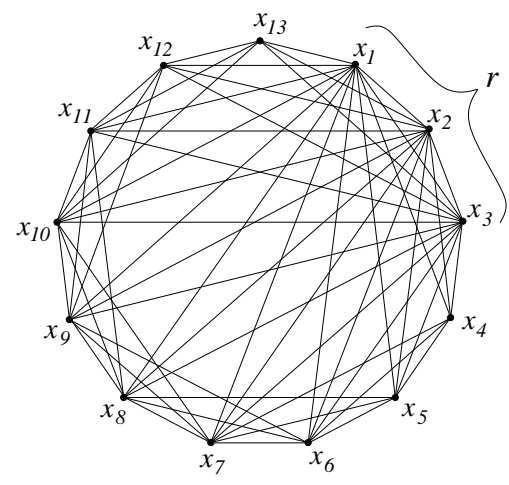

Figure 2. Construction showing that Theorem 3.4 is sharp $(n=13, r=4)$ 
Theorem 3.4. [CP92] The maximum number of edges of any r-quasi-planar convex geometric graph with $n \geq 2 r$ edges is

$$
2(r-1) n-\left(\begin{array}{c}
2 r-1 \\
2
\end{array}\right) \text {. }
$$

Theorem 3.5. (Valtr [V98]) Every $r$-quasi-planar $x$-monotone topological graph with $n$ vertices has at most $C_{r} n \log n$ edges, for a suitable constant $C_{r}$ depending on $r$.

Theorem 3.6. [PSS96] For any $r \geq 4$, every r-quasi-planar simple topological graph $G$ with $n$ vertices has at most $C_{r} n(\log n)^{2(r-3)}$ edges, for a suitable constant $C_{r}$ depending only on $r$.

In Section 6, we will point out that Theorem 3.6 remains true even if we drop the assumption that $G$ is simple, i.e., two edges may cross more than once.

For 3-quasi-planar topological graphs we have a linear upper bound.

Theorem 3.7. [AAPPS97] Every 3-quasi-planar simple topological graph $G$ with $n$ vertices has at most $C n$ edges, for a suitable constant $C$.

In Section 7, we give a short new proof of the last theorem, showing that here, too, one can drop the assumption that no two edges cross more than once (i.e., that $G$ is simple). In this case, previously no bound better than $O\left(n^{3 / 2}\right)$ was known. Theorem 3.7 can also be extended in another direction: it remains true for every topological graph $G$ with no $r+2$ edges such that each of the first $r$ edges crosses the last two and the last two edges cross each other. Of course, the constant $C$ in the theorem now depends on $r$ [PRT02].

All theorems in this section provide (usually linear) upper bounds on the number of edges of topological graphs satisfying certain conditions. In each case, one may ask whether a stronger statement is true. Is it possible that the graphs in question can be decomposed into a small number planar graphs? For instance, the following stronger form of Theorem 3.7 may hold:

Conjecture 3.8. There is a constant $k$ such that the edges of every 3-quasi-planar topological graph $G$ can be colored by $k$ colors so that no two edges of the same color cross each other.

McGuinness [Mc00] proved that Conjecture 3.8 is true for simple topological graphs, provided that there is a closed Jordan curve crossing every edge of $G$ precisely once. The statement is also true for $r$-quasi-planar convex geometric graphs, for any fixed $r$ (see [K88], [KK97]).

\section{Generalized thrackles and their relatives}

Two edges are said to be adjacent if they share an endpoint. We say that a graph drawn in the plane is a generalized thrackle if any two edges meet an odd number of times, counting their common endpoints, if they have any. That is, a graph is a generalized thrackle if and only if it has no two adjacent edges that cross an odd number of times and no two non-adjacent edges that cross an even number of times. In particular, a generalized thrackle cannot have two non-adjacent edges that are disjoint. Although at first glance this property may appear to be the exact opposite of planarity, surprisingly, the two notions are not that different. In particular, for bipartite graphs, they are equivalent.

Theorem 4.1. [LPS97] A bipartite graph can be drawn in the plane as a generalized thrackle if and only if it is planar.

Using the fact that every graph $G$ has a bipartite subgraph with at least $|E(G)| / 2$ edges, we obtain that if a graph $G$ of $n$ vertices can be drawn as a generalized thrackle, then $|E(G)|=O(n)$. 
Theorem 4.2. (Cairns-Nikolayevsky [CN00]) Every generalized thrackle with $n$ vertices has at most $2 n-2$ edges. This bound is sharp.

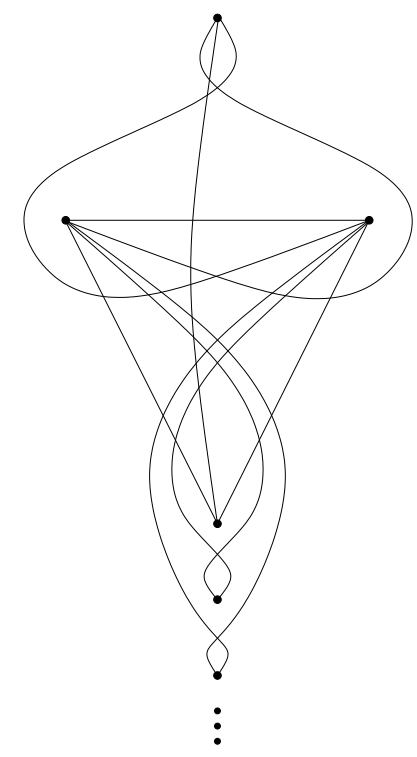

Figure 3. A generalized thrackle with $n$ vertices and $2 n-2$ edges

A geometric graph $G$ is a generalized thrackle if and only if it has no two disjoint edges. (The edges are supposed to be closed sets, so that two disjoint edges are necessarily non-adjacent.) One can relax this condition by assuming that $G$ has no $r$ pairwise disjoint edges, for some fixed $r \geq 2$. For $r=2$, it was proved by Hopf-Pannwitz [HP34] that every graph satisfying this property has at most $n$ edges, and that this bound is sharp. For $r=3$, the first linear bound on the number of edges of such graphs was established by Alon-Erdős [AE89], which was later improved to $3 n$ by GoddardKatchalski-Kleitman [GKK96]. For general $r$, the first linear bound was established in [PT94]. The best currently known estimate is the following:

Theorem 4.3. (Tóth [T00]) Every geometric graph with $n$ vertices and no $r$ pairwise disjoint edges has at most $2^{9}(r-1)^{2} n$ edges.

It is likely that the dependence of this bound on $r$ can be further improved to linear. If we want to prove the analogue of Theorem 4.3 for topological graphs, we have to make some additional assumptions on the structure of $G$, otherwise it is possible that any two edges of $G$ cross each other.

Conjecture 4.4. (Conway's Thrackle Conjecture) Let $G$ be a simple topological graph of $n$ vertices. If $G$ has no two disjoint edges, then $|E(G)| \leq n$.

For many related results, consult [LPS97], [CN00], [W71]. The next interesting open question is to decide whether the maximum number of edges of a simple topological graph with $n$ vertices and no three pairwise disjoint edges is $O(n)$.

\section{$5 \quad$ Locally planar graphs}

For any $r \geq 3$, a topological graph $G$ is called $r$-locally planar if $G$ has no selfintersecting path of length at most $r$. Roughly speaking, this means that the embedding of the graph is planar in a neighborhood of radius $r / 2$ around any vertex. In [PPTT02], we showed that there exist 3-locally planar geometric graphs with $n$ vertices and with at least constant times $n \log n$ edges. Somewhat 
surprisingly (to us), Tardos [T02] managed to extend this result to any fixed $r \geq 3$. He constructed a sequence of $r$-locally planar geometric graphs with $n$ vertices and a superlinear number of edges (approximately $n$ times the $\lfloor r / 2\rfloor$ times iterated logarithm of $n$ ). Moreover, these graphs are bipartite and all of their edges can be stabbed by the same line.

The following positive result is probably very far from being sharp.

Theorem 5.1. [PPTT02] The maximum number of edges of a 3-locally planar topological graph with $n$ vertices is $O\left(n^{3 / 2}\right)$.

For geometric graphs, much stronger results are known.

Theorem 5.2. [PPTT02] The maximum number of edges of a 3-locally planar x-monotone topological graph with $n$ vertices is $O(n \log n)$. This bound is asymptotically sharp.

For 5-locally planar $x$-monotone topological graphs, we have a slightly better upper bound on the number of edges: $O(n \log n / \log \log n)$. This bound can be further improved under the additional assumption that all edges of the graph cross the $y$-axis.

Theorem 5.3. [PPTT02] Let $G$ be an $x$-monotone $r$-locally planar topological graph of $n$ vertices all of whose edges cross the $y$-axis. Then, we have $|E(G)| \leq c n(\log n)^{1 /\lfloor r / 2\rfloor}$ for a suitable constant c.

\section{Strengthening Theorem 3.6}

In this section, we outline the proof of

Theorem 6.1. Every r-quasi-planar topological graph with $n$ vertices has at most

$$
f_{r}(n):=C_{r} n(\log n)^{4(r-3)}
$$

edges, where $r \geq 2$ and $C_{r}$ is a suitable positive constant depending on $r$.

Let $G$ be a graph with vertex set $V(G)$ and edge set $E(G)$. The bisection width $b(G)$ of $G$ is defined as the minimum number of edges, whose removal splits the graph into two roughly equal subgraphs. More precisely, $b(G)$ is the minimum number of edges running between $V_{1}$ and $V_{2}$, over all partitions of the vertex set of $G$ into two disjoint parts $V_{1} \cup V_{2}$ such that $\left|V_{1}\right|,\left|V_{2}\right| \geq|V(G)| / 3$. The pair-crossing number PAIR-CR $(G)$ of a graph $G$ is the minimum number of crossing pairs of edges in any drawing of $G$.

We need a recent result of Kolman and Matoušek [KM03], whose analogue for ordinary crossing numbers was proved in [PSS96] and [SV94].

Lemma 6.2. [KM03] Let $G$ be a graph of $n$ vertices with degrees $d_{1}, d_{2}, \ldots, d_{n}$. Then we have

$$
b^{2}(G) \leq c(\log n)^{2}\left(\operatorname{PAIR-CR}(G)+\sum_{i=1}^{n} d_{i}^{2}\right),
$$

where $c$ is a suitable constant.

We follow the idea of the original proof of Theorem 3.6. We establish Theorem 6.1 by double induction on $r$ and $n$. By Theorem 7.1 (in the next section), the statement is true for $r=3$ and for all $n$. It is also true for any $r>2$ and $n \leq n_{r}$, provided that $C_{r}$ is sufficiently large in terms of $n_{r}$, because then the stated bound exceeds $\left(\begin{array}{c}n \\ 2\end{array}\right)$. (The integers $n_{r}$ can be specified later so as to satify certain simple technical conditions.) 
Assume that we have already proved Theorem 6.1 for some $r \geq 3$ and all $n$. Let $n \geq n_{r+1}$, and suppose that the theorem holds for $r+1$ and for all topological graphs having fewer than $n$ vertices.

Let $G$ be an $(r+1)$-quasi-planar topological graph of $n$ vertices. For simplicity, we use the same letter $G$ to denote the underlying abstract graph. For any edge $e \in E(G)$, let $G_{e} \subset G$ denote the topological graph consisting of all edges of $G$ that cross $e$. Clearly, $G_{e}$ is $r$-quasi-planar. Thus, by the induction hypothesis, we have

$$
\operatorname{PAIR-CR}(G) \leq \frac{1}{2} \sum_{e \in E(G)}\left|E\left(G_{e}\right)\right| \leq \frac{1}{2}|E(G)| f_{r}(n) .
$$

Using the fact that $\sum_{i=1}^{n} d_{i}^{2} \leq 2|E(G)| n$ holds for every graph $G$ with degrees $d_{1}, d_{2}, \ldots, d_{n}$, Lemma 6.2 implies that

$$
b(G) \leq\left(c(\log n)^{2}|E(G)| f_{r}(n)\right)^{1 / 2} .
$$

Consider a partition of $V(G)$ into two parts of sizes $n_{1}, n_{2} \leq 2 n / 3$ such that the number of edges running between them is $b(G)$. Obviously, both subgraphs induced by these parts are $(r+1)$-quasiplanar. Thus, we can apply the induction hypothesis to obtain

$$
|E(G)| \leq f_{r+1}\left(n_{1}\right)+f_{r+1}\left(n_{2}\right)+b(G) .
$$

Comparing the last two inequalities, the result follows by some routine calculation.

\section{Strengthening Theorem 3.7}

The aim of this section is to prove the following stronger version of Theorem 3.7.

Theorem 7.1. Every 3-quasi-planar topological graph with $n$ vertices has at most $C n$ edges, for a suitable constant $C$.

Let $G$ be a 3-quasi-planar topological graph with $n$ vertices. Redraw $G$, if necessary, without creating 3 pairwise crossing edges so that the number of crossings in the resulting topological graph $\tilde{G}$ is as small as possible. Obviously, no edge of $\tilde{G}$ crosses itself, otherwise we could reduce the number of crossings by removing the loop. Suppose that $\tilde{G}$ has two distinct edges that cross at least twice. A region enclosed by two pieces of the participating edges is called a lens. Suppose there is a lens $\ell$ that contains no vertex of $\tilde{G}$. Consider a minimal lens $\ell^{\prime} \subseteq \ell$, by containment. Notice that by swapping the two sides of $\ell^{\prime}$, we could reduce the number of crossings without creating any new pair of crossing edges. In particular, $\tilde{G}$ remains 3 -quasi-planar. Therefore, we can conclude that

Claim 1. Each lens of $\tilde{G}$ contains a vertex.

We may assume without loss of generality that the underlying abstract graph of $G$ is connected, because otherwise we can prove Theorem 7.1 by induction on the number of vertices. Let $e_{1}, e_{2}, \ldots, e_{n-1} \in E(G)$ be a sequence of edges such that $e_{1}, e_{2}, \ldots, e_{i}$ form a tree $T_{i} \subseteq G$ for every $1 \leq i \leq n-1$. In particular, $e_{1}, e_{2}, \ldots, e_{n-1}$ form a spanning tree of $G$.

First, we construct a sequence of crossing-free topological graphs (trees), $\tilde{T}_{1}, \tilde{T}_{2}, \ldots, \tilde{T}_{n-1}$. Let $\tilde{T}_{1}$ be defined as a topological graph of two vertices, consisting of the single edge $e_{1}$ (as was drawn in $\tilde{G}$ ). Suppose that $\tilde{T}_{i}$ has already been defined for some $i \geq 1$, and let $v$ denote the endpoint of $e_{i+1}$ that does not belong to $T_{i}$. Now add to $\tilde{T}_{i}$ the piece of $e_{i+1}$ between $v$ and its first crossing with $\tilde{T}_{i}$. More precisely, follow the edge $e_{i+1}$ from $v$ up to the point $v^{\prime}$ where it hits $\tilde{T}_{i}$ for the first time, and denote this piece of $e_{i+1}$ by $\tilde{e}_{i+1}$. If $v^{\prime}$ is a vertex of $\tilde{T}_{i}$, then add $v$ and $\tilde{e}_{i+1}$ to $\tilde{T}_{i}$ and let $\tilde{T}_{i+1}$ be the resulting topological graph. If $v^{\prime}$ is in the interior of an edge $e$ of $\tilde{T}_{i}$, then introduce a new vertex at $v^{\prime}$. It divides $e$ into two edges, $e^{\prime}$ and $e^{\prime \prime}$. Add both of them to $\tilde{T}_{i}$, and delete $e$. Also add $v$ and $\tilde{e}_{i+1}$, and let $\tilde{T}_{i+1}$ be the resulting topological graph. 
After $n-2$ steps, we obtain a topological tree $\tilde{T}:=\tilde{T}_{n-1}$, which (1) is crossing-free, (2) has fewer than $2 n$ vertices, (3) contains each vertex of $\tilde{G}$, and (4) has the property that each of its edges is either a full edge, or a piece of an edge of $\tilde{G}$.

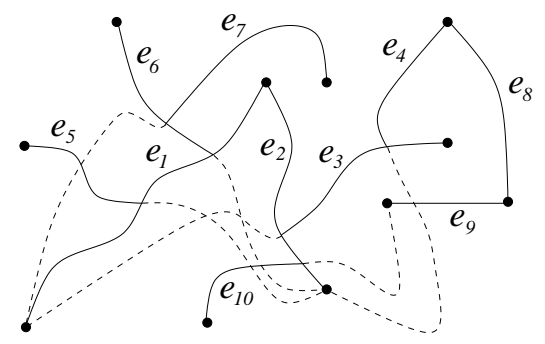

$T$

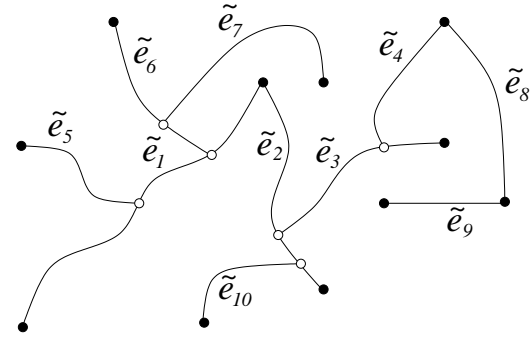

$\tilde{T}$

Figure 4. Constructing $\tilde{T}$ from $T$

Let $D$ denote the open region obtained by removing from the plane every point belonging to $\tilde{T}$. Define a convex geometric graph $H$, as follows. Traveling around the boundary of $D$ in clockwise direction, we encounter two kinds of different "features": vertices and edges of $\tilde{T}$. Represent each such feature by a different vertex $x_{i}$ of $H$, in clockwise order in convex position. Note that the same feature will be represented by several $x_{i}$ 's: every edge will be represented twice, because we visit both of its sides, and every vertex will be represented as many times as its degree in $\tilde{T}$. It is not hard to see that the number of vertices $x_{i} \in V(H)$ does not exceed $8 n$.

Next, we define the edges of $H$. Let $E$ be the set of edges of $\tilde{G} \backslash T$. Every edge $e \in E$ may $\operatorname{cross} \tilde{T}$ at several points. These crossing points divide $e$ into several pieces, called segments. Let $S$ denote the set of all segments of all edges $e \in E$. With the exception of its endpoints, every segment $s \in S$ runs in the region $D$. The endpoints of $s$ belong to two features along the boundary of $D$, represented by two vertices $x_{i}$ and $x_{j}$ of $H$. Connect $x_{i}$ and $x_{j}$ by a straight-line edge of $H$. Notice that $H$ has no loops, because if $x_{i}=x_{j}$, then, using the fact that $\tilde{T}$ is connected, one can easily conclude that the lens enclosed by $s$ and by the edge of $\tilde{T}$ corresponding to $x_{i}$ has no vertex of $G$ in its interior. This contradicts Claim 1.

Of course, several different segments may give rise to the same edge $x_{i} x_{j} \in E(H)$. Two such segments are said to be of the same type. Observe that two segments of the same type cannot cross. Indeed, as no edge intersects itself, the two crossing segments would belong to distinct edges $e_{1}, e_{2} \in E$. Since any two vertices of $G$ are connected by at most one edge, at least one of $x_{i}$ and $x_{j}$ corresponds to an edge (and not to a vertex) of $\tilde{T}$, which together with $e_{1}$ and $e_{2}$ would form a pairwise intersecting triple of edges, contradicting our assumption that $G$ is 3-quasi-planar.

Claim 2. $H$ is a 3-quasi-planar convex geometric graph.

To establish this claim, it is sufficient to observe that if two edges of $H$ cross each other, then the "features" of $\tilde{T}$ corresponding to their endpoints alternate in the clockwise order around the boundary of $D$. Therefore, any three pairwise crossing edges of $H$ would correspond to three pairwise crossing segments, which is a contradiction.

A segment $s$ is said to be shielded if there are two other segments, $s_{1}$ and $s_{2}$, of the same type, one on each side of $s$. Otherwise, $s$ is called exposed. An edge $e \in E$ is said to be exposed if at least one of its segments is exposed. Otherwise, $e$ is called a shielded edge.

In view of Claim 2, we can apply Theorem 3.4 [CP92] to $H$. We obtain that $|E(H)| \leq 4|V(H)|-$ $10<32 n$, that is, there are fewer than $32 n$ different types of segments. There are at most two exposed segments of the same type, so the total number of exposed segments is smaller than $64 n$, and this is also an upper bound on the number of exposed edges in $E$.

It remains to bound the number of shielded edges in $E$. 
Claim 3. There are no shielded edges.

Suppose, in order to obtain a contradiction, that there is a shielded edge $e \in E$. Orient $e$ arbitrarily, and denote its segments by $s_{1}, s_{2}, \ldots, s_{m} \in S$, listed according to this orientation. For any $1 \leq i \leq m$, let $t_{i} \in S$ be the (unique) segment of the same type as $s_{i}$, running closest to $s_{i}$ on its left side.

Since there is no self-intersecting edge and empty lens in $\tilde{G}$, the segments $t_{i}$ and $t_{i+1}$ belong to the same edge $f \in E$, for every $i<m$ (see Fig. 5). However, this means that both endpoints of $e$ and $f$ coincide, which is impossible.

We can conclude that $E$ has fewer than $64 n$ elements, all of which are exposed. Thus, taking into account the $n-1$ edges of the spanning tree $T$, the total number of edges of $\tilde{G}$ is smaller than $65 n$.
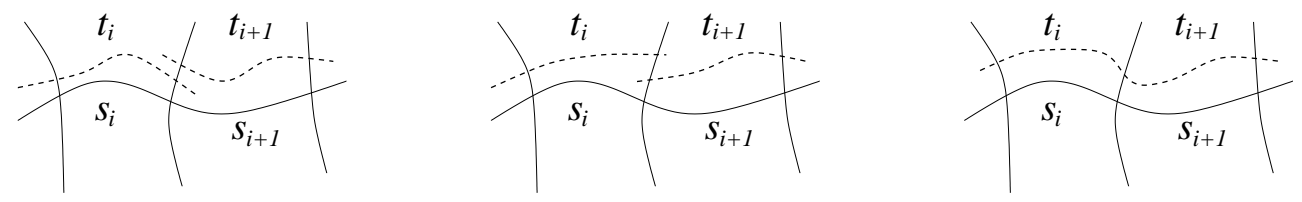

Figure 5. $t_{i}$ and $t_{i+1}$ belong to the same edge

\section{References}

[AAPPS97] P. K. Agarwal, B. Aronov, J. Pach, R. Pollack, and M. Sharir, Quasi-planar graphs have a linear number of edges, Combinatorica 17 (1997), 1-9.

[ACNS82] M. Ajtai, V. Chvátal, M. Newborn, and E. Szemerédi, Crossing-free subgraphs, in: Theory and Practice of Combinatorics, North-Holland Math. Stud. 60, North-Holland, Amsterdam-New York, 1982, $9-12$.

[AE89] N. Alon and P. Erdős, Disjoint edges in geometric graphs, Discrete Comput. Geom. 4 (1989), 287290.

[BT98] Bollobás and A. Thomason, Proof of a conjecture of Mader, Erdős and Hajnal on topological complete subgraphs, European J. Combin. 19 (1998), 883-887.

[BKV02] P. Braß, G. Károlyi, and P. Valtr, A Turán-type extremal theory for convex geometric graphs, in: Discrete and Computational Geometry - The Goodman-Pollack Festschrift (B. Aronov et al., eds.), Springer Verlag, Berlin, 2003, to appear.

[CN00] G. Cairns and Y. Nikolayevsky, Bounds for generalized thrackles, Discrete Comput. Geom. 23 (2000), 191-206.

[CP92] V. Capoyleas and J. Pach, A Turán-type theorem on chords of a convex polygon, Journal of Combinatorial Theory, Series B 56 (1992), 9-15.

[GKK96] W. Goddard, M. Katchalski, and D. J. Kleitman, Forcing disjoint segments in the plane, European J. Combin. 17 (1996), 391-395.

[HP34] H. Hopf and E. Pannwitz, Aufgabe Nr. 167, Jahresbericht der deutschen Mathematiker-Vereinigung 43 (1934), 114.

[KM03] P. Kolman and J. Matoušek, Crossing number, pair-crossing number, and expansion, manuscript.

[KSz96] J. Komlós and E. Szemerédi, Topological cliques in graphs II, Combin. Probab. Comput. 5 (1996), $79-90$.

[K84] A. V. Kostochka, Lower bound of the Hadwiger number of graphs by their average degree, Combinatorica 4 (1984), 307-316.

[K88] A. V. Kostochka, Upper bounds on the chromatic number of graphs (in Russian), Trudy Inst. Mat. (Novosibirsk), Modeli i Metody Optim., 10 (1988), 204-226.

[KK97] A. V. Kostochka and J. Kratochvíl, Covering and coloring polygon-circle graphs, Discrete Math. 163 (1997), 299-305.

[L84] F. T. Leighton, New lower bound techniques for VLSI, Math. Systems Theory 17 (1984), 47-70.

[LPS97] L. Lovász, J. Pach, and M. Szegedy, On Conway's thrackle conjecture, Discrete and Computational Geometry, 18 (1997), 369-376.

[M98] W. Mader, $3 n-5$ edges do force a subdivision of $K_{5}$, Combinatorica 18 (1998), 569-595. 
[Mc00] S. McGuinness, Colouring arcwise connected sets in the plane I, Graphs \& Combin. 16 (2000), 429-439.

[P99] J. Pach, Geometric graph theory, in: Surveys in Combinatorics, 1999 (J. D. Lamb and D. A. Preece, eds.), London Mathematical Society Lecture Notes 267, Cambridge University Press, Cambridge, 1999, $167-200$.

[PPST02] J. Pach, R. Pinchasi, M. Sharir, and G. Tóth, Topological graphs with no large grids, to appear. [PPTT02] J. Pach, R. Pinchasi, G. Tardos, and G. Tóth, Geometric graphs with no self-intersecting path of length three, in: Graph Drawing (M. T. Goodrich, S. G. Kobourov, eds.), Lecture Notes in Computer Science, 2528, Springer-Verlag, Berlin, 2002, 295-311.

[PRT02] J. Pach, R. Radoičić, and G. Tóth, On quasi-planar graphs, in preparation.

[PRTT02] J. Pach, R. Radoičić, G. Tardos, and G. Tóth, Graphs drawn with at most 3 crossings per edge, to appear.

[PSS96] J. Pach, F. Shahrokhi, and M. Szegedy, Applications of the crossing number, Algorithmica 16 (1996), 111-117.

[PT97] J. Pach and G. Tóth, Graphs drawn with few crossings per edge, Combinatorica 17 (1997), 427-439.

[PT94] J. Pach and J. Törőcsik, Some geometric applications of Dilworth's theorem, Discrete Comput. Geom. 12 (1994), 1-7.

[PR02] R. Pinchasi and R. Radoičić, On the number of edges in geometric graphs with no self-intersecting cycle of length 4, to appear.

[RST93] N. Robertson, P. Seymour, and R. Thomas, Hadwiger's conjecture for $K_{6}$-free graphs, Combinatorica 13 (1993), 279-361.

[SV94] O. Sýkora and I. Vrt́o, On VLSI layouts of the star graph and related networks, Integration, The VLSI Journal 17 (1994), 83-93.

[T02] G. Tardos, On the number of edges in a geometric graph with no short self-intersecting paths, in preparation.

[T84] A. Thomason, An extremal function for contractions of graphs, Math. Proc. Cambridge Philos. Soc. 95 (1984), 261-265.

[T00] G. Tóth, Note on geometric graphs, J. Combin. Theory, Ser. A 89 (2000), 126-132.

[T01] A. Thomason, The extremal function for complete minors, J. Combin. Theory Ser. B 81 (2001), 318-338.

[V98] P. Valtr, On geometric graphs with no $k$ pairwise parallel edges, Discrete and Computational Geometry 19 (1998), 461-469.

[W71] D. R. Woodall, Thrackles and deadlock, in: Combinatorial Mathematics and its Applications (Proc. Conf., Oxford, 1969), Academic Press, London, 1971, 335-347. 